\title{
Supersymmetric WZ-Poisson sigma model and twisted generalized complex geometry
}

\author{
Iván Calvo* \\ Department of Theoretical Physics \\ University of Zaragoza, E-50009 Zaragoza, Spain
}

\begin{abstract}
It has been shown recently that extended supersymmetry in twisted firstorder sigma models is related to twisted generalized complex geometry in the target. In the general case there are additional algebraic and differential conditions relating the twisted generalized complex structure and the geometrical data defining the model. We study in the Hamiltonian formalism the case of vanishing metric, which is the supersymmetric version of the WZPoisson sigma model. We prove that the compatibility conditions reduce to an algebraic equation, which represents a considerable simplification with respect to the general case. We also show that this algebraic condition has a very natural geometrical interpretation. In the derivation of these results the notion of contravariant connections on twisted Poisson manifolds turns out to be very useful.
\end{abstract}

*e-mail: icalvo@unizar.es 


\section{Introduction}

Generalized complex structures $([8,[7])$ on a manifold $M$ are objects defined on $T M \oplus T^{*} M$ which unify complex and symplectic geometry and interpolate between them. Recently, there has been much activity related to field theory realizations of generalized complex structures. Recall that extended supersymmetry in two-dimensional sigma models is related to complex geometry ([ $[$ ] $)$ in the target. On the other hand, in the first-order formulation the fields of the models take values in $T M \oplus T^{*} M$. This led to investigate the conditions for the existence of extended supersymmetry in first-order actions. The task was started in [10] and systematically carried out in [1], where it was shown that extended supersymmetry is closely related to generalized complex geometry in the target.

Later, in [15], it was shown that the Hamiltonian formalism allows to rederive and extend the results of [11] in a transparent fashion. In particular, it is proved in a model-independent way that if the generators of a second supersymmetry (denoted by $\mathbf{Q}_{2}(\epsilon)$ ) are to satisfy the supersymmetry algebra, the target must be a (twisted, if a WZ term is present) generalized complex manifold. Choosing a concrete model (i.e. a concrete Hamiltonian) and imposing that $\mathbf{Q}_{2}(\epsilon)$ be a symmetry should yield some compatibility conditions relating the geometrical objects defining the model and the generalized complex structure $\mathcal{J}$. In the general case ([1] $)$ these conditions include a number of algebraic as well as differential equations.

Following the approach of [15] we work out these compatibility conditions for the case of vanishing metric in the first-order sigma model, including a WZ term. When the closed 3 -form $H$ defining this twisting term vanishes, it is the supersymmetric version of the Poisson sigma model ([12]), related to deformation quantization $([4,2])$. For $H \neq 0$ it is the supersymmetric version of the WZ-Poisson sigma model (9]), whose target is equipped with a twisted Poisson structure $\Pi$. We show that the notion of contravariant connections on twisted Poisson manifolds is the key for unraveling the differential compatibility conditions between $\Pi$ and $\mathcal{J}$. We prove that, remarkably, the differential compatibility conditions are implied by the algebraic ones ${ }^{1}$, for which we also give a simple geometrical interpretation. The main result of this paper can be stated as follows:

The WZ-Poisson sigma model admits $N=2$ supersymmetry if and only if

$$
\mathcal{J}\left(L_{\Pi}\right) \subset L_{\Pi}
$$

where $L_{\Pi} \subset T M \oplus T^{*} M$ is the Dirac structure associated to $\Pi$ (see definitions in Section 3).

\footnotetext{
${ }^{1}$ This was already pointed out for the untwisted Poisson sigma model in 1 .
} 


\section{Contravariant connections on twisted Poisson man- ifolds}

Let $M$ be an $m$-dimensional manifold. A twisted Poisson structure or $H$-Poisson structure on $M(9,[13])$ is defined by a bivector field $\Pi$ and a closed 3 -form $H$ which satisfy a modified Jacobi identity:

$$
\Pi^{\mu \rho} \partial_{\rho} \Pi^{\nu \sigma}+\Pi^{\nu \rho} \partial_{\rho} \Pi^{\sigma \mu}+\Pi^{\sigma \rho} \partial_{\rho} \Pi^{\mu \nu}=\Pi^{\mu \alpha} \Pi^{\nu \beta} \Pi^{\sigma \gamma} H_{\alpha \beta \gamma}
$$

where $\partial_{\mu}:=\frac{\partial}{\partial x^{\mu}}$ and $x^{\mu}$ are local coordinates on $M$. Of course, in the untwisted case, $H=0, \Pi$ is a Poisson structure and (2.1) reduces to the Jacobi identity.

Assume we want to endow the twisted Poisson manifold $(M, \Pi, H)$ with a covariant connection. It is natural to demand this connection to be compatible with $\Pi$, so that the covariant derivative of $\Pi$ vanishes. Already in the untwisted case one can prove (14]) that a compatible covariant connection exists if and only if the rank of the Poisson tensor is constant. Much of the interest of Poisson and twisted Poisson structures resides in the fact that the rank of $\Pi$ can be non-constant, differing in an essential way from symplectic and twisted symplectic manifolds. Therefore, the notion of covariant derivative is not appropriate for twisted Poisson manifolds. The relevant concepts in Poisson manifolds are those of contravariant derivatives introduced by Vaisman ([14]) and contravariant connections developed by Fernandes ([5] $)$. Next, we extend their definitions and some results to the case of twisted Poisson manifolds.

A contravariant derivative on a vector bundle $E$ over the twisted Poisson manifold $(M, \Pi, H)$ is an operator $\nabla$ such that for each $\alpha \in \Omega^{1}(M), \nabla_{\alpha}$ maps sections of $E$ to sections of $E$ satisfying:

(i) $\nabla_{\alpha_{1}+\alpha_{2}} \psi=\nabla_{\alpha_{1}} \psi+\nabla_{\alpha_{2}} \psi$

(ii) $\nabla_{\alpha}\left(\psi_{1}+\psi_{2}\right)=\nabla_{\alpha} \psi_{1}+\nabla_{\alpha} \psi_{2}$

(iii) $\nabla_{f \alpha} \psi=f \nabla_{\alpha} \psi$

(iv) $\nabla_{\alpha}(f \psi)=f \nabla_{\alpha} \psi+\Pi^{\sharp} \alpha(f) \psi$

where $\Pi_{p}^{\sharp}: T_{p}^{*} M \rightarrow T_{p} M$ is defined by contraction with the first index of $\Pi$.

A contravariant derivative defines a contravariant connection in an analogous way to the covariant case. We shall be interested in defining the contravariant derivative of tensor fields on $M$. To this end it is enough to have a contravariant derivative on $E=T^{*} M$. Take local coordinates $x^{\mu}$ on $M$ and define the Christoffel symbols $\Gamma_{\rho}^{\mu \nu}$ by

$$
\nabla_{\mathrm{d} x^{\mu}} \mathrm{d} x^{\nu}=\Gamma_{\rho}^{\mu \nu} \mathrm{d} x^{\rho}
$$


The contravariant derivative of a tensor field $K$ of type $(p, q)$ is given by

$$
\begin{aligned}
\nabla^{\sigma} K_{\nu_{1} \ldots \nu_{q}}^{\mu_{1} \ldots \mu_{p}} & =\Pi^{\sigma \rho} \partial_{\rho} K_{\nu_{1} \ldots \nu_{q}}^{\mu_{1} \ldots \mu_{p}}-\sum_{a=1}^{p} \Gamma_{\rho}^{\sigma \mu_{a}} K_{\nu_{1} \ldots \nu_{q}}^{\mu_{1} \ldots \rho \mu_{p}}+ \\
& +\sum_{b=1}^{q} \Gamma_{\nu_{b}}^{\sigma \rho} K_{\nu_{1} \ldots \rho \nu_{q}}^{\mu_{1} \ldots \mu_{p}}
\end{aligned}
$$

A tensor field $K$ is said parallel if $\nabla K=0$. The relevant result for us is given by the following

Theorem: Let $(M, \Pi, H)$ be a twisted Poisson manifold. Then, there exists a contravariant connection such that $\Pi$ is parallel.

Proof: Let $\left\{U_{i}\right\}$ be an open cover of $M$. Take local coordinates $x^{\mu}$ on $U_{i}$ and define

$$
\Gamma_{(i) \rho}^{\mu \nu}=\partial_{\rho} \Pi^{\mu \nu}-\frac{1}{2} \Pi^{\mu \alpha} \Pi^{\nu \beta} H_{\rho \alpha \beta}
$$

On $U_{i}$, $\Pi$ is parallel for the contravariant connection $\nabla_{(i)}$ with symbols $\Gamma_{(i)}$. If $\sum_{i} f_{i}=1$ is a partition of unity subordinated to the cover $\left\{U_{i}\right\}, \nabla=\sum_{i} f_{i} \nabla_{(i)}$ gives a contravariant connection on $M$ such that $\nabla \Pi=0$.

\section{The Courant bracket on $T M \oplus T^{*} M$. Twisted gener- alized complex structures and twisted Poisson struc- tures.}

Let $M$ be an $m$-dimensional differentiable manifold. Given a closed 3-form $H$ the $H$-twisted Courant bracket on $T M \oplus T^{*} M$ is defined by

$$
\left[X_{1}+\xi_{1}, X_{2}+\xi_{2}\right]_{H}=\left[X_{1}, X_{2}\right]+\mathcal{L}_{X_{1}} \xi_{2}-\mathcal{L}_{X_{2}} \xi_{1}-\frac{1}{2} \mathrm{~d}\left(i_{X_{1}} \xi_{2}-i_{X_{2}} \xi_{1}\right)+i_{X_{1}} i_{X_{2}} H
$$

for $X_{1}+\xi_{1}, X_{2}+\xi_{2} \in C^{\infty}\left(T M \oplus T^{*} M\right)$. It is skew-symmetric but not a Lie bracket since it does not satisfy the Jacobi identity. The bracket (3.1) makes $T M \oplus T^{*} M$ into a Courant algebroid.

An $H$-Dirac structure $L$ in $T M \oplus T^{*} M$ is a maximally isotropic subbundle of $T M \oplus T^{*} M$ with respect to the natural pairing

$$
\left\langle X_{1}+\xi_{1}, X_{2}+\xi_{2}\right\rangle=\xi_{1}\left(X_{2}\right)+\xi_{2}\left(X_{1}\right)
$$

and whose sections are closed under the $H$-twisted Courant bracket. 
$H$-Poisson structures can be defined in a nice way in this language. Namely, the graph of a bivector field $\Pi$,

$$
L_{\Pi}=\left\{\left(\Pi^{\sharp} \xi, \xi\right) \in T M \oplus T^{*} M \mid \xi \in T^{*} M\right\}
$$

is an $H$-Dirac structure if and only if $\Pi$ is an $H$-Poisson structure.

If $x^{\mu}$ are local coordinates on $M$ and take $\left(\partial_{\mu}, \mathrm{d} x^{\mu}\right)$ as a basis in the fibres of $T M \oplus T^{*} M$, the bilinear form (3.2) reads

$$
\mathcal{I}=\left(\begin{array}{cc}
0 & 1_{m} \\
1_{m} & 0
\end{array}\right)
$$

An almost generalized complex structure is a linear map $\mathcal{J}: T M \oplus T^{*} M \rightarrow$ $T M \oplus T^{*} M$ such that $\mathcal{J}^{t} \mathcal{I} \mathcal{J}=\mathcal{I}$ and $\mathcal{J}^{2}=-1_{2 m}$. For such $\mathcal{J}$ define $p_{ \pm}=$ $\frac{1}{2}\left(1_{2 m} \pm i \mathcal{J}\right)$. The almost generalized complex structure $\mathcal{J}$ is an $H$-twisted generalized complex structure if

$$
p_{\mp}\left[p_{ \pm}\left(X_{1}+\xi_{1}\right), p_{ \pm}\left(X_{2}+\xi_{2}\right)\right]_{H}=0, \forall X_{1}+\xi_{1}, X_{2}+\xi_{2} \in C^{\infty}\left(T M \oplus T^{*} M\right)
$$

In the coordinate basis $\left(\partial_{\mu}, \mathrm{d} x^{\mu}\right)$ we can write,

$$
\mathcal{J}=\left(\begin{array}{ll}
J & P \\
L & K
\end{array}\right)
$$

where $J: T M \rightarrow T M, P: T^{*} M \rightarrow T M, L: T M \rightarrow T^{*} M, K: T^{*} M \rightarrow T^{*} M$. The condition $\mathcal{J}^{t} \mathcal{I} \mathcal{J}=\mathcal{I}$ becomes

$$
J_{\nu}^{\mu}+K_{\mu}{ }^{\nu}=0, \quad P^{\mu \nu}=-P^{\nu \mu}, \quad L_{\mu \nu}=-L_{\nu \mu}
$$

whereas $\mathcal{J}^{2}=-1_{2 m}$ translates into

$$
\begin{aligned}
& J_{\nu}^{\mu}{ }_{\nu}{ }_{\lambda}{ }_{\lambda}+P^{\mu \nu} L_{\nu \lambda}=-\delta^{\mu}{ }_{\lambda} \\
& J_{\nu}^{\mu} P^{\nu \lambda}+P^{\mu \nu} K_{\nu}{ }^{\lambda}=0 \\
& K_{\mu}{ }^{\nu} K_{\nu}{ }^{\lambda}+L_{\mu \nu} P^{\nu \lambda}=-\delta^{\mu}{ }_{\lambda} \\
& K_{\mu}{ }_{\mu} L_{\nu \lambda}+L_{\mu \nu} J_{\lambda}^{\nu}=0
\end{aligned}
$$

We shall not need the coordinate expression of the differential conditions coming from (3.5). We just want to point out that one of these conditions implies that $P$ is a Poisson structure, i.e.

$$
P^{\mu \rho} \partial_{\rho} P^{\nu \sigma}+P^{\nu \rho} \partial_{\rho} P^{\sigma \mu}+P^{\sigma \rho} \partial_{\rho} P^{\mu \nu}=0
$$


The action of a 2 -form $b$ on $T M \oplus T^{*} M$ defined by

$$
e^{b}(X+\xi):=X+\xi+i_{X} b
$$

is said a $b$-transform. The interesting point is that this action is a morphism of Courant algebroids. Concretely,

$$
e^{b}\left(\left[X_{1}+\xi_{1}, X_{2}+\xi_{2}\right]_{H}\right)=\left[e^{b}\left(X_{1}+\xi_{1}\right), e^{b}\left(X_{2}+\xi_{2}\right)\right]_{H+\mathrm{d} b}
$$

It follows that the $b$-transform of an $H$-twisted generalized complex structure $\mathcal{J}$

$$
\mathcal{J}_{b}:=e^{b} \mathcal{J} e^{-b}
$$

is an $(H+\mathrm{d} b)$-twisted generalized complex structure.

Under a $b$-transform (3.10), the $H$-Dirac structure $L_{\Pi}$ is transformed into $e^{b}\left(L_{\Pi}\right)$, which is an $(H+\mathrm{d} b)$-Dirac structure. However, the subbundle $e^{b}\left(L_{\Pi}\right)$ is the graph of a bivector field if and only if $1_{m}+b \Pi$ is invertible. In this case, $e^{b}\left(L_{\Pi}\right)=L_{\Pi_{b}}$, where

$$
\Pi_{b}:=\Pi\left(1_{m}+b \Pi\right)^{-1}
$$

is an $(H+\mathrm{d} b)$-Poisson structure.

If $b$ is closed the transformation (3.10) gives an orthogonal automorphism of the twisted Courant bracket. The most general orthogonal automorphism of the twisted Courant bracket is a semidirect product of a diffeomorphism of $M$ and a $b$-transform with $b \in \Omega_{\text {closed }}(M)$.

\section{Extended supersymmetry in the WZ-Poisson sigma model}

Let $S^{1,1}$ be the supercircle with coordinates $\bar{\sigma}=(\sigma, \theta)$. In local coordinates the cotangent bundle of the superloop space, $T^{*} \mathbf{L} M$, is given by scalar superfields $\Phi^{\mu}(\sigma, \theta)$ and spinorial superfields $S_{\mu}(\sigma, \theta)$. In components,

$$
\Phi^{\mu}(\sigma)=X^{\mu}(\sigma)+\theta \lambda^{\mu}(\sigma), \quad S_{\mu}(\sigma)=\rho_{\mu}(\sigma)+\theta p_{\mu}(\sigma)
$$

where $X^{\mu}$ and $p_{\mu}$ are bosonic fields.

Assume that $M$ is equipped with a closed 3-form $H$. Then, the following 2-form on $T^{*} \mathbf{L} M$ is symplectic:

$$
\omega=\int_{S^{1,1}} \mathrm{~d} \sigma \mathrm{d} \theta\left(\delta \Phi^{\mu} \wedge \delta S_{\mu}-\frac{1}{2} H_{\mu \nu \rho} D \Phi^{\mu} \delta \Phi^{\nu} \wedge \delta \Phi^{\rho}\right)
$$

where $\delta$ stands for the de Rham differential and $D=\partial_{\theta}-\theta \partial_{\sigma}$. Since $\omega$ is closed and non-degenerate, it defines a Poisson bracket on functions of the superfields which we shall denote by $\{\cdot, \cdot\}$. 
The basic Poisson brackets read:

$$
\begin{aligned}
& \left\{\Phi^{\mu}(\bar{\sigma}), \Phi^{\nu}\left(\bar{\sigma}^{\prime}\right)\right\}=0 \\
& \left\{\Phi^{\mu}(\bar{\sigma}), S_{\nu}\left(\bar{\sigma}^{\prime}\right)\right\}=\delta_{\nu}^{\mu} \delta\left(\bar{\sigma}-\bar{\sigma}^{\prime}\right) \\
& \left\{S_{\mu}(\bar{\sigma}), S_{\nu}\left(\bar{\sigma}^{\prime}\right)\right\}=H_{\mu \nu \rho} D \Phi^{\rho} \delta\left(\bar{\sigma}-\bar{\sigma}^{\prime}\right)
\end{aligned}
$$

with $\delta\left(\bar{\sigma}-\bar{\sigma}^{\prime}\right)$ the superspace delta distribution. Notice that

$$
S_{\mu} \mapsto S_{\mu}-B_{\mu \nu} D \Phi^{\nu}
$$

is a canonical transformation for closed $B$.

The Hamiltonian formulation of the $N=1$ supersymmetric WZ-Poisson sigma model (WZ-PSM) is as follows. The phase space of the theory, denoted by $\mathcal{C}$, is the set of points of $T^{*} \mathbf{L} M$ satisfying the constraints:

$$
D \Phi^{\mu}(\sigma, \theta)+\Pi^{\mu \nu}\left(\Phi^{\mu}\right) S_{\nu}(\sigma, \theta)=0, \mu=1, \ldots, m
$$

which are a consequence of the singular nature of the Lagrangian of the WZ-PSM ([9]). The Hamiltonian of the WZ-PSM can be written:

$$
\mathcal{H}=\int_{S^{1,1}} F_{\mu}(\sigma, \theta)\left(D \Phi^{\mu}(\sigma, \theta)+\Pi^{\mu \nu}\left(\Phi^{\mu}\right) S_{\nu}(\sigma, \theta)\right) \mathrm{d} \sigma \mathrm{d} \theta
$$

where the fields $F_{\mu}$ act as Lagrange multipliers. The consistency of the model requires $\Pi$ to be an $H$-Poisson structure. In the Hamiltonian formalism this is obtained as the condition for the dynamics to preserve the submanifold $\mathcal{C}$, i.e. $\left.\left\{D \Phi^{\mu}+\Pi^{\mu \nu} S_{\nu}, \mathcal{H}\right\}\right|_{\mathcal{C}}=0$.

By construction, the WZ-PSM is invariant under the supersymmetry transformation generated by:

$$
\mathbf{Q}_{1}(\epsilon)=\int_{S^{1}} \mathrm{~d} \sigma \epsilon\left(p_{\mu} \lambda^{\mu}-\rho_{\mu} \partial X^{\mu}-\frac{1}{3} H_{\mu \nu \rho} \lambda^{\mu} \lambda^{\nu} \lambda^{\rho}\right)
$$

where $\partial X^{\mu}=\frac{\partial X^{\mu}}{\partial \sigma}$.

We address now the issue of extended supersymmetry in the WZ-PSM. The most general ansatz for a second supersymmetry transformation is given by ([15])

$$
\mathbf{Q}_{2}(\epsilon)=-\frac{1}{2} \int_{S^{1,1}} \mathrm{~d} \sigma \mathrm{d} \theta \epsilon\left(2 D \Phi^{\mu} S_{\nu} J_{\mu}^{\nu}+D \Phi^{\mu} D \Phi^{\nu} L_{\mu \nu}+S_{\mu} S_{\nu} P^{\mu \nu}\right)
$$

As shown in [15] in a model-independent way, the generators $\mathbf{Q}_{2}(\epsilon)$ satisfy the supersymmetry algebra if and only if

$$
\mathcal{J}=\left(\begin{array}{cc}
J & P \\
L & -J^{t}
\end{array}\right)
$$


is an $H$-twisted generalized complex structure. In this context, the canonical transformation (4.4) is identified with a $b$-transform for closed $B$, i.e. an automorphism of the Courant algebroid. If $B$ is not closed, $S_{\mu} \mapsto S_{\mu}-B_{\mu \nu} D \Phi^{\nu}$ is not a canonical transformation and it changes the twisting term in (4.2). In particular, if $H=\mathrm{d} B$ it untwists the symplectic structure.

It remains to find out when $\mathbf{Q}_{2}(\epsilon)$ generates a symmetry transformation of the WZ-PSM. That is, when

$$
\left.\left\{\mathbf{Q}_{2}(\epsilon), \mathcal{H}\right\}\right|_{\mathcal{C}}=0
$$

At this stage one expects that (4.10) holds only if some compatibility conditions relating $\mathcal{J}$ and $\Pi$ are satisfied. These conditions were worked out in the Lagrangian formalism for a general (untwisted) first-order sigma model in [1]. It turns out that in the general case some algebraic as well as differential conditions must be imposed. Our aim is to prove that in the WZ-PSM the differential conditions are automatically implied by the algebraic ones. We shall see that the contravariant connections introduced in Section 2 are extremely helpful in the derivation of this result.

A direct calculation shows that (4.10) holds if and only if the following two conditions are met:

Algebraic condition:

$$
P^{\mu \nu}+J_{\rho}^{\mu} \Pi^{\rho \nu}+\Pi^{\mu \rho} J_{\rho}^{\nu}-\Pi^{\mu \rho} L_{\rho \sigma} \Pi^{\sigma \nu}=0
$$

Differential condition:

$$
\begin{aligned}
& \frac{1}{2}\left[\Pi^{\mu \beta} \partial_{\beta} P^{\nu \rho}-\partial_{\beta} \Pi^{\mu \nu} P^{\beta \rho}-\partial_{\beta} \Pi^{\mu \rho} P^{\nu \beta}+\right. \\
& +\left(\Pi^{\mu \beta} \partial_{\beta} J_{\gamma}^{\nu}-\partial_{\beta} \Pi^{\mu \nu} J_{\gamma}^{\beta}+\partial_{\gamma} \Pi^{\mu \beta} J_{\beta}^{\nu}\right) \Pi^{\gamma \rho}+ \\
& +\Pi^{\nu \gamma}\left(\Pi^{\mu \beta} \partial_{\beta} J_{\gamma}^{\rho}-\partial_{\beta} \Pi^{\mu \rho} J_{\gamma}^{\beta}+\partial_{\gamma} \Pi^{\mu \beta} J_{\beta}{ }_{\beta}\right)- \\
& \left.-\Pi^{\nu \gamma}\left(\Pi^{\mu \beta} \partial_{\beta} L_{\gamma \alpha}+\partial_{\gamma} \Pi^{\mu \beta} L_{\beta \alpha}+\partial_{\alpha} \Pi^{\mu \beta} L_{\gamma \beta}\right) \Pi^{\alpha \rho}\right]+ \\
& +\left(P^{\alpha \nu}+J_{\tau}^{\alpha} \Pi^{\tau \nu}\right) \Pi^{\mu \gamma} \Pi^{\beta \rho} H_{\beta \alpha \gamma}=0
\end{aligned}
$$

The untwisted version of the differential condition (4.12) was deduced in [1] (see equation (6.35) therein) for a general untwisted sigma model. In the case of non-vanishing metric it was interpreted as a condition of constancy with respect to a covariant derivative compatible with the metric tensor. The WZ-PSM is the limit of vanishing metric of the twisted first-order sigma model and there is no such covariant derivative at hand. We have learnt in Section 2 that the natural objects which allow to compare tangent spaces at different points of a twisted Poisson manifold are contravariant connections. In fact, after some manipulations 
we can rewrite condition (4.12) in terms of the local symbols $(2.4)^{2}$ :

$$
\begin{aligned}
& \nabla^{\mu} P^{\nu \rho}+\left(\nabla^{\mu} J_{\gamma}^{\nu}\right) \Pi^{\gamma \rho}+\Pi^{\nu \gamma}\left(\nabla^{\mu} J_{\gamma}^{\rho}\right)-\Pi^{\nu \gamma}\left(\nabla^{\mu} L_{\gamma \alpha}\right) \Pi^{\alpha \rho}+ \\
& +\left(P^{\alpha \nu}+J_{\tau}^{\alpha} \Pi^{\tau \nu}-J^{\nu}{ }_{\tau} \Pi^{\tau \alpha}-\Pi^{\alpha \delta} L_{\delta \tau} \Pi^{\tau \nu}\right) \Pi^{\mu \gamma} \Pi^{\beta \rho} H_{\beta \alpha \gamma}=0
\end{aligned}
$$

Using that $\nabla \Pi=0$ we can go one step further and write:

$$
\begin{aligned}
& \nabla^{\mu}\left(P^{\nu \rho}+J_{\gamma}^{\nu} \Pi^{\gamma \rho}+\Pi^{\nu \gamma} J_{\gamma}^{\rho}-\Pi^{\nu \gamma} L_{\gamma \alpha} \Pi^{\alpha \rho}\right)+ \\
& +\left(P^{\alpha \nu}+J^{\alpha}{ }_{\tau} \Pi^{\tau \nu}-J^{\nu} \Pi^{\tau \alpha}-\Pi^{\alpha \delta} L_{\delta \tau} \Pi^{\tau \nu}\right) \Pi^{\mu \gamma} \Pi^{\beta \rho} H_{\beta \alpha \gamma}=0
\end{aligned}
$$

which is obviously satisfied if the algebraic condition (4.11) holds. Hence, we obtain the remarkable result that the WZ-PSM has a second supersymmetry (4.8) if and only if $\mathcal{J}$ is a generalized complex structure and the algebraic condition (4.11) is satisfied. This gives an enormous simplification with respect to the general sigma model, in which the differential condition analogous to (4.12) is not necessarily implied by the algebraic ones.

Now, we would like to give a geometrical interpretation of $(4.11)^{3}$, which imposes a compatibility condition between the $H$-Poisson structure $\Pi$ and the Poisson structure $P$. Define two endomorphisms of $T M \oplus T^{*} M$ by

$$
\tau_{1}:=\left(\begin{array}{cc}
0 & \Pi \\
0 & 1_{m}
\end{array}\right), \quad \tau_{2}:=\left(\begin{array}{cc}
1_{m} & -\Pi \\
0 & 0
\end{array}\right)
$$

Notice that $\tau_{i}^{2}=\tau_{i}, i=1,2 . \tau_{1}$ projects onto the Dirac structure $L_{\Pi}$ (see definition (3.3)) along $T M . \quad \tau_{2}$ projects onto $T M$ along $L_{\Pi}$. In particular, $\operatorname{Im}\left(\tau_{1}\right)=\operatorname{Ker}\left(\tau_{2}\right)=L_{\Pi}$, which will be the important point for us.

In matrix notation the condition (4.11) can be expressed as

$$
\tau_{2}\left(\begin{array}{cc}
J & P \\
L & -J^{t}
\end{array}\right) \tau_{1}=0
$$

which says that $\mathcal{J}$ can be restricted to an endomorphism of $L_{\Pi}$. That is, the WZ-PSM supports extended supersymmetry if and only if

$$
\mathcal{J}\left(L_{\Pi}\right) \subset L_{\Pi}
$$

Note that the canonical transformation (4.4) can be viewed as a $b$-transform with $b=-B \in \Omega_{\text {closed }}(M)$ acting on $\mathcal{J}$ and $\Pi$. Since a canonical transformation does not modify the Poisson brackets, one would expect that (4.11) hold for the transformed objects

$$
\mathcal{J}_{-B}=\left(\begin{array}{cc}
J_{-B} & P_{-B} \\
L_{-B} & -J_{-B}^{t}
\end{array}\right), \quad \Pi_{-B}=\Pi\left(1_{m}-B \Pi\right)^{-1}
$$

\footnotetext{
${ }^{2}$ We omit the subscript $(i)$ referring to an open set of a cover of $M$.

${ }^{3}$ The condition (4.11) was already deduced in [1], but a geometrical interpretation was lacking.
} 
This is not evident from (4.11). However, the result follows easily by using that the expression (4.16) is manifestly invariant under a $b$-transform. But the argument is purely algebraic so that the result holds even if $B$ is not closed.

The fact that in the WZ-PSM there is only one algebraic compatibility condition between $\mathcal{J}$ and $\Pi$, given by (4.11), should make easier the search of backgrounds admitting extended supersymmetry. Notice that when trying to solve (4.11) one can use that it is invariant under a $b$-transform. Therefore, if $H=\mathrm{d} B$ and $1_{m}-B \Pi$ is invertible one can look for solutions of (4.11) in terms of the untwisted objects and twist back at the end of the day.

\section{Conclusions}

We have continued the work [15], where it was shown that extended supersymmetry in phase space for twisted two-dimensional sigma models requires a twisted generalized complex structure $\mathcal{J}$ on the target. We have considered the particular case of the supersymmetric WZ-Poisson sigma model (WZ-PSM) and analysed the compatibility conditions relating $\mathcal{J}$ and the twisted Poisson structure $\Pi$. The notion of contravariant connections on twisted Poisson manifolds helps to show that the WZ-PSM admits $N=2$ supersymmetry if and only if

$$
\mathcal{J}\left(L_{\Pi}\right) \subset L_{\Pi}
$$

where $L_{\Pi} \subset T M \oplus T^{*} M$ is the Dirac structure associated to $\Pi$. The simplification coming from the absence of differential conditions makes the WZ-PSM into an interesting mathematical laboratory and should allow to construct examples of supersymmetric backgrounds not previously discussed in the literature.

In this paper we have not considered the model defined on a surface with boundary. First, it would be worth to study in detail the admissible branes for the non-supersymmetric WZ-PSM, which should yield a twisted extension of the results of $[3]$. When $H=\mathrm{d} B$ and $1_{m}-B \Pi$ is invertible, one can untwist the theory and reduce it to the standard Poisson sigma model. However, for nonexact $H$ or non-invertible $1_{m}-B \Pi$ there are subtle aspects which deserve to be investigated. Then, one could turn to the supersymmetric WZ-PSM to identify the D-branes which preserve supersymmetry.

Of course, it would be interesting to perform the calculations presented in this paper for a completely general twisted first-order sigma model, i.e. with non-vanishing metric. This will be the subject of further research.

Acknowledgments: I thank Fernando Falceto for many insightful remarks and for a careful reading of the manuscript. I am also grateful to Izu Vaisman for important comments on the proof of the theorem of Section 2. This work was supported by MEC (Spain), grant FPU and grant FPA2003-02948. 


\section{References}

[1] L. Bergamin, Generalized complex geometry and the Poisson Sigma Model, Mod. Phys. Lett. A 20 (2005), 985-996, arXiv:hep-th/0409283.

[2] I. Calvo and F. Falceto, Star products and branes in Poisson-Sigma models, arXiv:hep-th/0507050.

[3] — Poisson reduction and branes in Poisson-Sigma models, Lett. Math. Phys. 70 (2004), 231-247, arXiv:hep-th/0405176.

[4] A. S. Cattaneo and G. Felder, A path integral approach to the Kontsevich quantization formula, Commun. Math. Phys. 212 (2000), 591-611, arXiv: math.QA/9902090

[5] R. L. Fernandes, Connections in Poisson Geometry I. Holonomy and Invariants, J. Differential Geom. 54 (2000), 303-365, arXiv:math.DG/0001129.

[6] S. J. Gates, C. M. Hull, and M. Rocek, Twisted multiplets and new supersymmetric nonlinear sigma models, Nucl. Phys. B 248 (1984), 157.

[7] M. Gualtieri, Generalized complex geometry, Oxford University DPhil thesis, arXiv:math.DG/0401221.

[8] N. Hitchin, Generalized Calabi-Yau manifolds, Quart.J.Math.Oxford Ser. 54 (2003), 281-308, arXiv:math.DG/0209099

[9] C. Klimcik and T. Strobl, WZW-Poisson manifolds, J. Geom. Phys. 43 (2002), 341-344, arXiv:math.SG/0104189.

[10] U. Lindstrom, Generalized $N=(2,2)$ Supersymmetric Non-Linear Sigma Models, Phys. Lett. B 587 (2004), 216-224, arXiv:hep-th/0401100.

[11] U. Lindstrom, R. Minasian, A. Tomasiello, and M. Zabzine, Generalized complex manifolds and supersymmetry, Commun. Math. Phys. 257 (2005), 235-256, arXiv:hep-th/0405085.

[12] P. Schaller and T. Strobl, Poisson structure induced (topological) field theories, Mod. Phys. Lett. A9 (1994), 3129-3136, arXiv:hep-th/9405110.

[13] P. Severa and A. Weinstein, Poisson geometry with a 3-form background, Prog. Theor. Phys. Suppl. 144 (2001), 145, arXiv:math.SG/0107133.

[14] I. Vaisman, Lectures on the geometry of Poisson manifolds, Birkhäuser, Basel-Boston-Berlin, 1994.

[15] M. Zabzine, Hamiltonian perspective on generalized complex structure, arXiv:hep-th/0502137 